\title{
o wushu como uma ferramenta para o desenvolvimento motor, cognitivo e socioafetivo na escola: um estudo exploratório
}

\author{
Everton de Souza DA SILVA ${ }^{1}$, Diego Pereira ALVES ${ }^{1}$, Lucas Ribeiro DOS SANTOS ${ }^{1}$, Marcelo \\ Augusto P. dos SANTOS ${ }^{1}$, Sumara Brito SOARES ${ }^{1}$, Bianca MIARKA ${ }^{2}$ \\ ${ }^{1}$ Universidade Bandeirante Anhanguera, Campus Campo Limpo (Brazil) \\ ${ }^{2}$ Universidade Federal de Juiz de Fora, Campus Governador Valadares (Brazil)
}

Recepción: 26/12/2016; Aceptación: 13/07/2017; Publicación: 16/07/2017.

\begin{abstract}
Resumo professores; sociologia.

\section{El wushu como herramienta para el desarrollo motor, cognitivo y socioafectivo en la escuela: un estudio exploratorio}

O presente trabalho investigou a utilização do wushu como ferramenta para o desenvolvimento motor, cognitivo e socioafetivo na escola entre professores de Educação Física (PEF) e comparou praticantes e não praticantes em conceito, compreensão e na aplicação de práticas ressignificadas no Ensino Fundamental I e II em São Paulo, no Brasil. Para isso, participaram $40 \mathrm{PEF}$, separados em dois grupos Praticantes de wushu (PW, $n=27$ ) e Não Praticantes de wushu (NPW, $n=13$ ). Todos responderam um questionário com 11 perguntas objetivas sobre a prática de wushu por escolares, com respostas em quatro escalas ("não atende", "parcialmente", "plenamente" e "supera"). Para comparar as respostas utilizou-se o teste de Mann Whitney, $p \leq 0,05$. 0 resultado mostrou diferença entre os grupos, na qual PW consideram a modalidade um sistema válido para ensinar aspectos relacionados com as artes marciais para escolares, enquanto NPW acredita ser pouco eficiente. Ambos os grupos indicaram o wushu, como uma atividade que atende e supera as necessidades de desenvolvimento motor, cognitivo e socioafetivo em escolares. Em contrapartida, cerca de $30 \%$ dos NPW indicou que o wushu é difícil para ser utilizado em escolares e $40 \%$ desse grupo informou que as práticas de wushu durante a formação na graduação foram apenas parcialmente ou não foram realizadas de maneira clara. Esses resultados sugerem a possibilidade de haver relação entre a experiência dos professores na promoção de determinadas modalidades, podendo estar o ensino das artes marciais dependente do fato dos professores serem ou não praticantes.

Palavras-chave: Artes marciais; desportos de combate; Educação Física; educação; currículo; treinamento de

\section{Resumen}

El presente estudio investigó la utilización del wushu en la escuela por profesores de Educación Física (FEP) como herramienta para el desarrollo motor, cognitivo y socioafectivo del alumnado, y comparó a profesores practicantes y no practicantes en concepto, comprensión y aplicación de prácticas resignificadas de wushu en los grados I y II Enseñanza Primaria en São Paulo, Brasil. Para este fin, 40 PEF fueron asignados a un grupo de dos, practicantes wushu (PW, $n=27$ ) o no practicantes de wushu (NPW, n=13). Todos los participantes respondieron a un cuestionario compuesto por 11 preguntas objetivas sobre la práctica de wushu por parte de los escolares, con respuestas divididas en cuatro niveles ("no asiste", "parcialmente", "completamente" y "excede"). Se utilizó la prueba de Mann Whitney para comparar las respuestas, $p \leq 0,05$. Los resultados mostraron diferencias entre los grupos, considerando los PW que el wushu es un contenido válido para enseñar a los escolares aspectos relacionados con las artes marciales, mientras que los NPW lo consideraron poco eficiente. Ambos grupos identificaron al wushu como una actividad que satisface y supera las necesidades de desarrollo motor, cognitivo y socioafectivo de los escolares. Por otro lado, alrededor del

\section{Wushu as a tool for motor, cognitive and socioaffective development at school: an exploratory study}

\section{Abstract}

The present work investigated the use of wushu at school by Physical Education teachers (PEF) as a tool for the motor, cognitive and socioaffective development, and compared practitioners and nonpractitioners in concept, understanding and application of wushu resignified practices in Elementary School grades I and II in São Paulo, Brazil. For this purpose, 40 PEFs were assigned to one of two groups, wushu Practitioners (PW, $n=27$ ) or Non wushu Practitioners (NPW, $n=13$ ). All participants answered a questionnaire composed of 11 objective questions about the wushu practice by schoolchildren, with answers ranging on four levels ("does not attend", "partially", "fully" and "exceeds"). The Mann Whitney test was used to compare the responses, $p \leq 0.05$. Results showed differences between groups, in which PW considered wushu a valid content to teach aspects related to martial arts for schoolchildren, while NPW believed to be inefficient. Both groups identified wushu as an activity that meets and exceeds the needs of motor, cognitive and socioaffective development in schoolchildren. On the other hand, about $30 \%$ of NPWs

*E-mail: evertonsilva@usp.br 
$30 \%$ de los NPW indicaron que el wushu es difícil de utilizar en el contexto escolar, y el $40 \%$ de los NPW consideraron que las prácticas de wushu recibidas durante su formación inicial fueron escasas o inexistentes. Estos resultados sugieren la posibilidad de que exista una relación entre la experiencia de los profesores y la promoción de ciertas prácticas, y que la enseñanza de las artes marciales puede depender de la propia condición de practicantes de los profesores de Educación Física.

Palabras clave: Artes marciales; deportes de combate; Educación Física; educación; currículum; formación de profesores; sociología. indicated that wushu is difficult to use in schoolchildren, and $40 \%$ of NPWs reported that wushu practices during initial teacher training were only partially or not sufficiently taught. These results suggest the possibility of a relationship between the teachers' experience and the promotion of certain practices, depending the teaching of martial arts on the teachers' practical experience.

Keywords: Martial arts; combat sports; Physical Education; education; curriculum; teachers training; sociology.

\section{Introdução}

No Brasil, o ensino fundamental concerne ao período em que crianças e adolescentes, com idade entre 6 e 14 anos, frequentam o meio escolar e, consequentemente, as aulas de Educação Física (Brasil, 1997). Apesar das práticas de artes marciais e esportes de combate, chamadas de AM\&EC (Cynarski \& Lee-Barron, 2014; Del Vecchio \& Franchini, 2006; Pérez-Gutiérrez, Gutiérrez-García, \& Escobar-Molina, 2011), terem sido oficialmente introduzidas nas aulas de Educação Física escolar há quase vinte anos (Brasil, 1997), muito embora já fossem exploradas no meio escolar desde o início do século XX em escolas militares (Alves Junior, 2006; Mocarzel \& Murad, 2013), ainda trazem consigo questões sociais eminentes sobre a sua importância no desenvolvimento motor, cognitivo e socioafetivo tanto no Brasil (Gomes, Barros, Freitas, Rufino \& Darido, 2013), quanto em outros lugares do mundo (Johnson \& Ha, 2015; Kusnierz, Cynarski, \& Gorner 2017). Sabe-se que o preconceito sobre a introdução do processo de ensino-aprendiagem das AM\&EC em meio escolar está associado com a preocupação relacionada ao aumento de ações agressivas (Mocarzel \& Murad, 2013; Ortega \& Antunes, 2013; Robles, 2008). Porém, já existem indicativos da prevenção advinda da prática de AM\&EC contra a violência (Di Zio, 2010; Hortiguela, Gutiérrez-García, \& Hernando-Garijo, 2017; Menéndez \& Fernández-Río, 2016; Tejero-González, Balsalobre-Fernández, \& Ibáñez-Cano, 2011). Outro motivo pelo qual as AM\&EC não serem ministradas com frequência durante as aulas de Educação Física escolar concerne à falta de conhecimento ou, ainda à não atenção em relação a tais práticas, ainda minoritárias em meios escolares brasileiros (Mocarzel \& Murad, 2013). Particularmente, AM tradicionais, como o wushu, são ministradas esporadicamente ou negligenciadas (Cynarski \& Lee-Barron, 2014; Mocarzel, Ferreira, \& Murad, 2014; Zivin, et al., 2001). Não se sabe ao certo qual a percepção dos professores de Educação Física sobre a prática de wushu no ensino fundamental brasileiro e porque ainda existe essa lacuna na aprendizagem das AM\&EC.

O wushu engloba diversos tipos de práticas chinesas (Theeboom, Dong, \& Vertonghen, 2012), as quais permitem desenvolver habilidades motoras, cognitivas, socioafetivas e estimulam a autovalorização do praticante (Martins, 2011; Theeboom, De Knop, \& Vertonghen, 2009). Como exemplo dessas práticas existem o tai chi chuan e chi kung (formas de meditação), a manutenção de posturas básicas, prática de taolus (formas coreografadas) com ou sem implementos, exercícios de sanshou ou sanda (técnicas de percussão, como socos e chutes) e atividades de shuaijiao (técnicas de quedas, agarre, desequilíbrio e projeções). Essas práticas estão atreladas ao conceito de motricidade fina (Alves, 2012), pois desenvolvem coordenação visuomanual, utilizando mãos e pés para realizar movimentos com precisão com/sem objetos. Assim como desenvolve a motricidade global, na qual ações específicas da modalidade, deslocamentos e ritmos são melhorados (Almeida, 2009). A modalidade desenvolve o equilíbrio (estático e dinâmico), base para ações da prática de formas coreografadas, assim como para realização de técnicas de percussão, quedas, agarre, desequilíbrio e projeções (Alves, 2012). Quanto ao desenvolvimento do esquema corporal, a imagem do corpo no wushu se organiza a partir da associação entre o corpo e o ambiente (Alves, 2012). Outra característica do desenvolvimento motor nessa AM é a noção espacial a partir da percepção da posição do próprio corpo em um determinado espaço e a organização temporal desenvolvida em crianças e adolescentes (Almeida, 2009). A lateralidade está em função da percepção de simetria do corpo e contribui para determinar a estrutura espacial, o eixo do próprio 
corpo e a relação desse eixo com o meio ambiente (Alves, 2012). Além disso, existe o estudo do wude (preceitos filosóficos) (Theeboom et al., 2012). Apesar desse leque de possibilidades, pouco se sabe sobre os processos de ensino-aprendizagem de wushu ao longo do ensino escolar por parte dos professores de Educação Física e, se a formação extracurricular com a prática de wushu de tais professores influencia no ensino escolar de wushu, como um dos temas trabalhados em AM\&EC.

A perspectiva teórica para esta pesquisa é inspirada pela teoria humanista das artes marciais, as quais apresentam as AM\&EC como atividade de desenvolvimento psicofísico-social (Cynarski, et al., 2017). De acordo com essa linha, EC é conceituado como uma variação das artes marciais e é distinguido das mesmas por promoverem confrontos competitivos (Cynarski \& Skowron 2014), enquanto as AM são definidas como "uma categoria histórica de métodos de luta desarmada ou com uso de armas brancas combinadas com elementos filosóficos e/ou religiosos" (Cynarski \& Skowron, 2014). Nessa concepção, o termo lutas é utilizado quando existe disputa em que dois, ou mais, adversários utilizam técnicas e estratégias de desequilíbrio, contusão, imobilização ou exclusão, de um território de combate, caracterizando-se por regras específicas que punem atitudes desleais no desenvolvimento das ações (Brasil, 1997; Cynarski, et al., 2017). No Brasil, uma das AM mais difundidas é a arte marcial chinesa, nomeada wushu, que mostra grande aumento de praticantes desde sua introdução no país, durante a década de 1960 (Acevedo, Gutiérrez-García, \& Cheung, 2011; Lima, 2000; Mocarzel et al., 2014; Pérez-Gutiérrez \& Gutiérrez García, 2012; Torres, 2011). De modo geral, não existem muitas pesquisas sobre a produção acadêmica em relação ao ensino-aprendizagem de AM\&EC nas escolas (Barros \& Gabriel, 2011; Correia \& Franchini, 2010; Rufino \& Darido, 2015), especialmente, sobre o wushu, o universo acadêmico parece ser ainda mais restrito (Mocarzel \& Murad, 2013), mas é possível identificar inúmeros eventos científicos para a divulgação de resultados de pesquisas com essa temática (Franchini \& Del Vecchio, 2012; Martin, 2015). Portanto, investigar a prática de wushu dentro do ensino escolar pode contribuir para área de estudo em pedagogia das AM\&EC (Barros \& Gabriel, 2011; Correia \& Franchini, 2010; Gomes, 2008; Millán, 2015; Rufino \& Darido, 2015).

A filosofia tradicional do wushu como AM, é inclusa na prática através de uma atitude condicionada para melhora da saúde mental e física e dos efeitos positivos relacionados com aumento de eficácia e capacidade (Bu, Haijun, Yong, Chaohiu, Xiaoyuan, \& Singh, 2010; Tarabanov, 2011). Particularmente, o wushu obteve expansão da sua prática em meios pedagógicos nos países do Ocidente porque diz respeito não só à luta em si, mas também ao seu significado educacional conectado aos valores chineses associados ao simbolismo natural e com o princípio da existência do ying e yang, ou seja, da interação entre duas forças opostas (Theeboom \& Knop, 1999; Verthonghen \& Theeboom, 2010; Theeboom et al., 2012; Weng, 1990). A partir dessa particularidade, parte-se do pressuposto que o wushu possa contribuir na formação de crianças e adolescentes do ensino fundamental através de práticas que envolvam manifestações filosóficas e éticas de maneira lúdica, o que pode ser importante em termos de preservação da cultura e história corporal nas AM\&EC (Amorim, 2004; Cynarski \& Lee-Barron, 2014; Franchini \& Del Vecchio, 2012; Mocarzel \& Murad, 2013; Rufino \& Darido, 2015; Zivin et al., 2001).

Por parte dos professores, a resiliência em utilizar práticas de wushu ou de outras AM, consideradas complexas para serem ministradas durante as aulas escolares, tem afetado a inclusão plena das AM\&EC nos currículos da Educação Física escolar brasileira (Nascimento \& Almeida, 2007; Rufino \& Darido, 2015). Entrevistas e questionários ao redor do mundo mostram resultados irregulares sobre a inclusão de AM, com valores culturais e filosóficos próprios, em âmbito escolar. No Japão, as escolas utilizam o Budo (AM japonesas) conectado com a prática de AM\&EC, como meio de obter benefícios motores, cognitivos e socioafetivos ao longo do processo educacional (Sasaki, 2006). Em contrapartida, as pesquisas realizadas no continente europeu mostram que os países que utilizam AM\&EC preferem integrar atividades de auto-defesa, judô e karate (Theeboom \& Knop, 1999; Villamón, Gutiérrez-García, Espartero, \& Molina, 2005). Na Espanha, menos de 2\% dos escolares participaram de aulas com AM\&EC (Villamón et al., 2005). Enquanto os Estados Unidos não se preocupam em ensinar AM\&EC durante as aulas de Educação Física (Theeboom et al., 2012) e o wushu parece ser ensinado apenas em escolas comerciais (Theeboom et al., 2012). Apesar dessas importantes contribuições para ciência de AM\&EC, pouco se sabe sobre a prática de wushu ao longo do processo de ensino-aprendizagem em escolas do Ocidente. 
Portanto, o presente estudo tem como hipótese que existam professores que dimensionem a importância da prática de wushu para escolares, porém que não façam a aplicação dessa AM como proposta para o desenvolvimento motor, cognitivo e socioafetivo em crianças e adolescentes. A inclusão dessa prática nas aulas de Educação Física Escolar pode ser importante para modificação da conduta social através do ensino-aprendizagem de fatores advindos de elementos históricos e culturais próprios da modalidade, como disciplina, equilíbrio do corpo e da mente, persistência e respeito ao próximo - elementos base da filosofia chinesa (Mocarzel, 2011). Para tanto, o objetivo desse estudo é discutir o wushu como ferramenta para o desenvolvimento motor, cognitivo e socioafetivo em crianças e adolescentes na escola e verificar se existe diferença na compreensão e aplicação de práticas dessa AM no Ensino Fundamental I e II entre professores praticantes e não praticantes de wushu.

\section{Metodologia}

\subsection{Delineamento do estudo}

Esse é um estudo exploratório realizado em uma pesquisa de campo com professores de Educação Física escolar, comparando professores de Educação Física escolar praticantes e não praticantes de wushu. Utilizou-se um questionário objetivo com respostas em formato de escala (Thomas \& Nelson, 2002), para comparar a percepção dos profissionais sobre o uso da modalidade para o desenvolvimento motor, cognitivo e socioafetivo no Ensino Fundamental I e II em São Paulo, Brasil. Para desenvolvimento do questionário e coleta de dados, que divide a avaliação instrumental em quatro: i) Avaliação da qualidade da aula, como proposta para análise do desenvolvimento motor, cognitivo, socioafetivo com práticas de wushu por escolares; ii) Métricas externas da qualidade de ensino-aprendizagem do wushu; iii) Métricas internas da qualidade de ensinoaprendizagem do wushu, e; iv) Métricas de qualidade de ensino-aprendizagem em uso aplicado durante as aulas de Educação Física. Em um segundo momento foram feitas comparações entre os grupos através de análises estatísticas e discussões sobre os resultados.

\subsection{Participantes}

Participaram desse estudo 40 Professores de Educação Física escolar (PEF), separados em dois grupos, Praticantes de wushu (PW, $n=27$ ) e Não Praticantes de wushu (NPW, $n=13$ ) da Região Metropolitana de São Paulo, no Brasil, entre março e setembro de 2016. Ambos os grupos investigados trabalham com escolares (crianças e adolescentes) do Ensino Fundamental I e II do Brasil há mais de um ano, sendo que 63\% do grupo PW e 53\% do grupo NPW ministram aulas há mais de quatro anos. Em relação ao grupo PW, todos treinam a modalidade há mais de três anos, sendo que $92,6 \%$ é praticante há mais de quatro anos e faixa preta. Além disso, 33,3\% treinam por mais de dez horas semanais, 33,3\%, entre cinco e dez horas por $30 \%$ e os demais praticam entre quatro e cinco horas por semana. Dentre os participantes PW, 92,7\% competiram nos últimos quatro anos e 7,4\% da amostra nunca competiu. Para escolha da amostra, estabeleceu-se como critério de inclusão no grupo PW, PEF com um mínimo de três anos de prática regular de wushu com mais de quatro horas semanais. Como critério de exclusão, estabeleceu-se a ausência da Licenciatura em Educação Física pelos PEF dos grupos PW e NPW. Seguindo a resolução 196/96 do Conselho Nacional de Saúde, o respectivo trabalho faz parte de um estudo temático encaminhado e aprovado por um Comitê de Ética em Pesquisa com seres humanos da Universidade Anhanguera de Valinhos (007/2016), pelo que cada um dos participantes assinou um termo de consentimento livre e esclarecido para participação do estudo.

\subsection{Procedimento - desenvolvimento do questionário}

Desenvolvimento do questionário quanto ao conteúdo: As principais características que descrevem o questionário de acordo com um modelo de qualidade através das questões são baseadas em estudos prévios sobre iniciação e prática de AM\&EC no meio escolar (Avelar-Rosa, Gomes, Figueiredo \& López-Ros, 2015; Bueno, 2012; Cynarski \& Lee-Barron, 2014; Del Vecchio \& Franchini, 2012; Figueiredo, 1997, 1998, 2000; Gomes, 2008; Lima, 1997; Light, 2014; Nascimento \& Almeida, 2007; Pérez-Gutiérrez et al., 2011). 
Organização da estrutura do questionário: Para organizar o conteúdo de maneira objetiva e categorizada (i.e. funcionalidade, confiabilidade, usabilidade, eficiência, manutenção e portabilidade) e organizar o resultado de uma até quatro escalas, foi utilizado o modelo de normas de qualidade ISO/IEC 9126 para produtos, indicado por pesquisadores prévios (Ando, Miarka \& Pinto, 2016; Anjos \& Moura, 2016).

Aplicação e questionário: A aplicação do questionário desenvolvido foi realizada, de acordo com as diretrizes de estrutura e respectivos conteúdos, sobre o processo de ensino-aprendizagem, conectado com o desenvolvimento motor, cognitivo e socioafetivo. Para isso, perguntou-se sobre:

(1) Funcionalidade: As práticas do wushu atendem as necessidades de desenvolvimento motor, cognitivo e socioafetivo em crianças e adolescentes?

(2) Confiabilidade: A prática de wushu desenvolve os aspectos relacionados com desenvolvimento motor, cognitivo e socioafetivo de crianças e adolescentes no que é proposta, de maneira adequada aos Parâmetros Curriculares Nacionais (1997)?

(3) Usabilidade:A prática de wushu apresenta falhas no ensino-aprendizagem para escolares, se sim, com que frequência?

(4) Eficiência: De modo geral, você considera a prática de wushu um método fácil, em ensino-aprendizagem, para usar em escolares? É fácil entender o conceito e a aplicação a que se destina a prática de wushu? De modo geral, você considera o wushu um sistema eficiente, rápido e objetivo de ensinar aspectos condizentes as artes marciais para escolares?

(5) Manutenção e Portabilidade: 0 conhecimento sobre a prática de wushu é fácil para ser usado em escolares? As informações, sobre prática de wushu, durante a sua formação na graduação foram mostradas de maneira clara? De modo geral, você considera o wushu como uma arte marcial fácil de ser modificada e adaptada nas suas necessidades atuais/futuras no processo de ensino-aprendizagem para crianças e adolescentes? É possível usar o wushu para outras práticas de lutas e esportivas dentro da escola e ou academia que exijam mais espaço ou regras fechadas?

Durante a aplicação, o avaliador explicou para os participantes sobre cada parte do questionário. Na primeira parte, as diretrizes estão relacionadas à definição e ao uso de medidas para apurar a satisfação dos critérios de qualidade da aula, como proposta para análise do desenvolvimento motor, cognitivo, socioafetivo com práticas de wushu por escolares. A segunda parte visa medições externas, ou seja, o comportamento do sistema baseado no sistema de avaliação do serviço, para a mensuração de atributos de características gerais; a parte três engloba medições internas, que são providas do próprio professor, dessas mesmas características; e a parte quatro, trata de qualidade em uso das atividades, que para o professor, é o efeito da combinação das seis características de qualidade do serviço, ou seja, da aula, dimensionando o uso das práticas de atividade física em um contexto específico de utilização na prática de atividade física escolar (ABNT, NBR-ISO/IEC 9126-1, 2003).

\subsection{Análise estatística}

Os dados descritivos são apresentados em frequência e percentual. Para comparar os dados entre os grupos, utilizou-se o teste não paramétrico de Mann Whitney. Em todas as análises, foi considerado como nível de significância o valor de $5 \%(p<0,05)$. Utilizou-se o Programa Statistical Package for Social Sciences 20.0 (SPSS IBM๑, EUA).

\section{Resultados}

A tabela 1 mostra os resultados descritivos, em frequência e percentual, das respostas dos PEF separados em PW e NPW. 
Tabela 1. Resultados descritivos sobre as questões em conceito, compreensão e aplicação do wushu no Ensino Fundamental I e II por professores praticantes (PW) e não praticantes (NPW) da modalidade

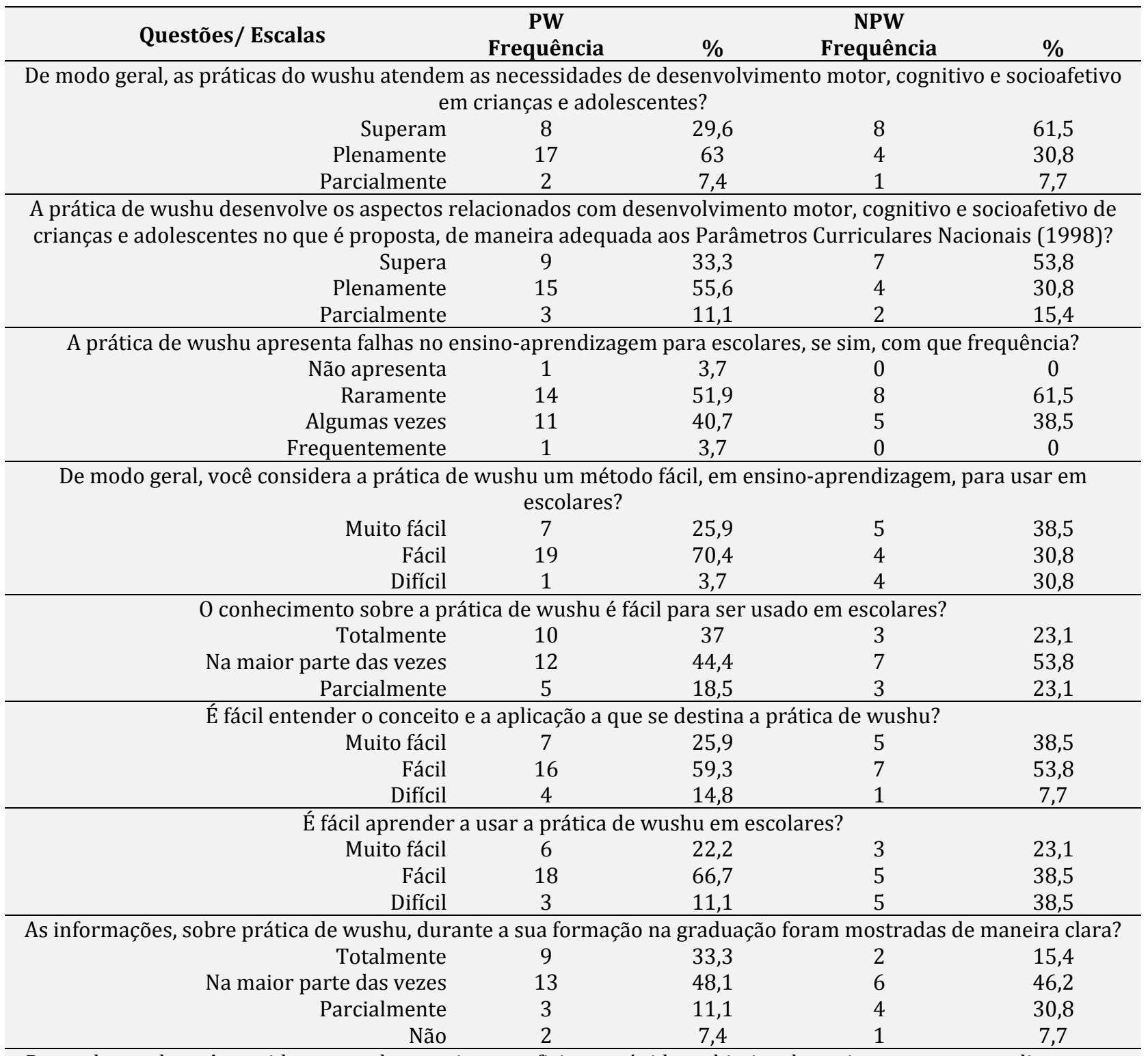

De modo geral, você considera o wushu um sistema eficiente, rápido e objetivo de ensinar aspectos condizentes as artes marciais para escolares?

$\begin{array}{rcccc}\text { Muito eficiente } & 11 & 40,7 & 0 & 0 \\ \text { Eficiente } & 15 & 55,6 & 8 & 61,5 \\ \text { Pouco eficiente } & 1 & 3,7 & 5 & 38,5\end{array}$

De modo geral, você considera o wushu como uma arte marcial fácil de ser modificada e adaptada nas suas necessidades atuais/futuras no processo de ensino-aprendizagem para crianças e adolescentes?

$\begin{array}{rcccc}\text { Totalmente } & 9 & 33,3 & 4 & 30,8 \\ \text { Na maior parte das vezes } & 11 & 40,7 & 6 & 46,2 \\ \text { Parcialmente } & 6 & 22,2 & 3 & 23,1 \\ \text { Não } & 1 & 3,7 & 0 & 0\end{array}$

É possível usar o wushu para outras práticas de lutas e esportivas dentro da escola e ou academia que exijam mais

\begin{tabular}{rccccc}
\multicolumn{5}{c}{ espaço ou regras fechadas? } \\
Totalmente & 15 & 55,6 & 8 & 61,5 \\
Na maior parte das vezes & 8 & 29,6 & 4 & 30,8 \\
Parcialmente & 4 & 14,8 & 1 & 7,7
\end{tabular}

Nota: Na primeira, na segunda, na quarta, quinta, sexta, sétima, nona e décima primeira questões as últimas escalas "não atende", "não faz", "muito difícil", "não", "muito difícil", "muito difícil", "ineficiente" e "não" não obtiveram 0 frequência de escolha, respectivamente

Nas comparações estatísticas sobre o ensino-aprendizagem de wushu por escolar para desenvolvimento motor, cognitivo e socioafetivo houve diferença significativa entre os grupos, o 
grupo PW considera a modalidade um sistema válido (muito eficiente/eficiente) para ensinar aspectos das artes marciais para escolares, enquanto o grupo NPW acredita ser pouco eficiente $(U=$ 70.500, $W=448.500, z=-3,420, p \leq 0,001)$. Não foram observadas outras diferenças entre PW e NPW ( $\mathrm{p}>0,05$ para todas as análises).

Todos os participantes do estudo responderam que recomendariam a utilização do wushu para escolares, dentre os professores participantes do estudo, cerca de 70,4\% do grupo PW acreditam ser necessário realizar adaptações para ambientes escolares, enquanto 53,8\% do NPW não acha necessário fazer modificações para prática de wushu em ambiente escolar.

\section{Discussão}

O objetivo desse estudo é discutir o wushu como ferramenta para o desenvolvimento motor, cognitivo e socioafetivo em crianças e adolescentes em meio escolar e verificar se existe diferença na compreensão e aplicação de práticas dessa AM no Ensino Fundamental I e II entre professores praticantes e não praticantes de wushu. 0 resultado mostra diferença entre os grupos, na qual praticantes de wushu consideram a modalidade um sistema válido para ensinar aspectos relacionados as AM para escolares, enquanto o grupo de professores não praticantes acredita ser pouco eficiente. Não praticantes e praticantes indicam que o wushu atende as necessidades de desenvolvimento motor, cognitivo e socioafetivo em crianças e adolescentes adotadas pelos Parâmetros Curriculares Nacionais. As práticas de wushu superam ou satisfazem tais necessidades e raramente apresentam falhas no ensino-aprendizagem para escolares. 0 conhecimento atrelado ao wushu parece ser fácil de ser transmitido aos escolares. Por sua vez, todos os participantes do estudo responderam que recomendariam a utilização do wushu para escolares, dentre os professores participantes do estudo, cerca de $70 \%$ dos praticantes acreditam ser necessário realizar adaptações para ambientes escolares, enquanto cerca de $50 \%$ dos não praticantes não acha necessário fazer modificações para prática de wushu em ambiente escolar. Em contrapartida, cerca de $30 \%$ dos não praticantes indicou ser um método difícil para ser utilizado em escolares e aproximadamente $40 \%$ dos não praticantes indicou que as informações sobre a prática de wushu durante a formação na graduação foram apenas parcialmente ou não foram realizadas de maneira clara.

Com relação à questão em que o wushu pode ou não ser considerado uma prática eficaz para ensinar aspectos gerais das AM, a diferença entre os grupos pode ser explicada pelo impacto do valor educacional do contexto da AM\&EC, que está fortemente condicionado pelo tipo, estilo e modelo instrucional conduzido pelo professor (Lima, 2012; Metzler, 2011). A resistência dos professores escolares para utilizar práticas do wushu ou de AM tradicionais pode estar relacionada com a rigidez e formalismo no processo de ensino-aprendizagem de mestres que vislumbram manter o processo pedagógico utilizado ao longo do século XX (Del Vecchio \& Franchini, 2012; Donohue, 2005; Durántez, 2004; Figueiredo, 2006). Nessa tradição, merecem destaque três características que entram em confronto com as indicações dos Parâmetros Curriculares Nacionais brasileiros (1997): 1) a detenção de poder e controle total do professor sobre o aluno, em interação mandatória e autoritária por parte do professor; 2) hierarquia contínua e submissão de acordo com o sistema de graduação da modalidade, e; 3) autoridade do Iemoto, com centralização de poder pelas federações e confederações (Saeki, 1994). Atualmente, o wushu e as AM\&EC, em geral, são adaptadas para iniciantes e escolares para aumentar o foco nos aspectos educacionais e no desenvolvimento de elementos cognitivos e motores, assim como para aumentar o aspecto colaborativo dos alunos, evitar lesões e reduzir competitividade exacerbada na aplicação prática (Diamond \& Lee, 2011; Miarka \& Conceição, 2012). Outras iintervenções com AM\&EC mostraram auxiliar o desenvolvimento de funções em crianças entre 4 e 12 anos (Diamond \& Lee, 2011). Essas adaptações pedagógicas, assim como a ocidentalização das AM, são indicadas para aulas específicas de wushu (Theeboom \& Knop, 1999).

No presente estudo, a ideia da eficácia do wushu, como espelho para ensino de AM em geral, pode ter advindo das concepções filosóficas da modalidade, possivelmente moduladas nos professores do grupo praticantes. Em recente trabalho, autores mostraram que praticantes recém ingressos no wushu valorizam pouco a filosofia atrelada à modalidade, sendo que $68 \%$ acreditam 
que as concepções filosóficas são pouco ou nada importantes (Mocarzel et al., 2014). Contudo, após um ano de treinamento, 92\% dos praticantes passaram a acreditar que a filosofia do wushu é importante para a prática (Mocarzel et al., 2014). Atualmente, discussões sobre a adaptação de elementos tradicionais ao público ocidental, ou seja, sobre a dialética do desenvolvimento/inovação em confronto com a cultural da herança/ancestralidade do wushu indicam um posicionamento da China a favor de adaptações da prática à cultura ocidental para que haja difusão dessa AM no mundo (Theeboom et al., 2012). Segundo estudo prévio, o objetivo operacional dessa modificação é integrar o caráter nacional dos valores chineses com a generalidade da cultura física ocidental (Theeboom et al., 2012).

Quanto ao desenvolvimento motor, a utilização do wushu parece atender as necessidades, quando observadas as respostas para as duas primeiras questões do presente estudo. Por sua vez, a adaptação da prática ao meio educacional, pode gerar resultados positivos (Theeboom, De Knop \& Weiss, 1995). Em três semanas de intervenção de campo com 112 crianças entre oito e 12 anos de idade, foram comparados o desempenho e o prazer da prática do wushu entre um programa tradicional de treinamento - baseado em um número limitado de práticas (sem equipamentos para iniciantes), realização e análise da técnica como resultado da tarefa, não participação na criação de atividades técnico-táticas e avaliação pelo resultado do desempenho técnico - versus um programa experimental voltado para maestria- baseado em variação de ações, uso imediato de equipamentos, exercícios de curta duração, criação de elementos técnico-táticos pelos participantes, desenvolvimento progressivo de habilidades e autoavaliação (Theeboom et al., 1995). Os resultados revelaram melhora motoras em ambos os grupos, porém as crianças do grupo experimentalmostraram níveis significativamente maiores de prazer e exibiram melhores habilidades motoras, quando comparadas com as orientadas para o desempenho da tarefa, no programa tradicional (Theeboom et al., 1995). Esses dados reforçam a indicação da adaptação dos métodos de ensino para crianças em razão da adaptação das práticas aumentar a motivação e, consequentemente, melhorar o desenvolvimento motor (Theeboom et al., 1995).

Apesar dessa eminente difusão (Theeboom et al., 2012), na presente pesquisa quase metade dos professores não praticantes de wushu indicaram que as informações sobre a prática da modalidade ao longo da formação na graduação foram mostradas parcialmente ou ausentes. Essa ausência ou a pouca aprendizagem em ensino superior, mostrados nas respostas dos entrevistados, reforçam os dados apresentados por Ferreira (2006), que indicam reduzida aplicação de ensinoaprendizagem das AM\&EC em meio escolar associado compouca prática regular ao longo do processo de formação do profissional no ensino superior, especialmente, de professores com formação antecedente a introdução das lutas nos Parâmetros Curriculares Nacionais (1997), os quais não possuíram na grade de ensino a disciplina de lutas e, mesmo assim, são considerados aptos pelo sistema educacional à ministrá-la em contexto do ensino escolar (Ferreira, 2006). Gomes e Avelar-Rosa (2012) compararam a quantidade de instituições de ensino em diferentes países e mostraram existir no Brasil apenas 24 instituições de ensino superior com curso de lutas, sendo que apenas uma ensinava wushu. Enquanto nações com menor número populacional, como Portugal, França e Espanha tinham entre 31 e 37 instituições com curso de lutas, porém as grades curriculares dessas universidades também não mostravam o wushu como proposta de ensino (Gomes \& Avelar-Rosa, 2012). Para tanto, no Brasil existem cursos de Pós-graduação Lato Sensu (especialização) em lutas, tais como os cursos: Lutas e Artes Marciais (Universidade Estadual de São Paulo - UNESP, 2016) Lutas e Artes Marciais: da pedagogia ao treinamento (Universidade Gama Filho - UGF, 2011), Metodologia e didática do ensino da Educação Física e das Artes Marciais (Faculdade Einstein - FACEI, 2016); com recorte para cursos de Pós-graduação Lato Sensu (especialização) em alguns estilos de wushu como é o caso do Tai Chi Chuan (Universidade de São Paulo - USP, 2016) e do Shuai Jiao (Faculdade Einstein - FACEI, 2015). Do ponto de vista institucional, a robustez do wushu pode ser vista através da integração da International wushu Federationao SportAccord, Federação Internacional das Federações Desportivas (IWUF, 2015) e, por isso, participa dos Combat Games (Jogos com chancela do Comitê Olímpico Internacional, que conectam todas as AM\&EC reconhecidas pela própria instituição). A IWUF faz parte do COI, apesar de não estar no programa dos Jogos Olímpicos (IWUF, 2012).

Outra possibilidade sobre os resultados encontrados, estaria no fato de que em ambiente escolar, as AM\&EC sofrem um preconceito muito grande, porque, socialmente, sua prática está 
vinculada a espetáculos com violência e à agressividade, conforme indicam estudos prévios (Ortega \& Antunes, 2013; Menéndez \& Fernández-Río, 2016; Mocarzel \& Murad, 2013; Robles, 2008). Especula-se que outra questão pode ser referente ao fato de que as AM\&EC foram predominantemente associadas ao sexo masculino ao longo da história (Miarka, Marques \& Franchini, 2011), isso pode interferir diretamente na prática por escolares do sexo feminino. Particularmente, questões de gênero nas AM\&EC são evidentes em âmbito escolar e, no caso do wushu, não tem sido estudadas com a atenção que merecem. Cabem aqui duas questões para futuros estudos: i) em que medida é necessário adaptar a prática de uma maneira diferenciada para meninos e/ou meninas? e ii) o quanto há de necessidade em adaptar AM, como o wushu, aos requerimentos e expectativas dessas práticas em relação à redução de violência entre escolares? Naturalmente, essas e outras questões sociais das práticas das AM\&EC são abordadas nas aulas de Educação Física escolar, destacando-se a importância social da abordagem das AM, que mostraram melhorar a responsabilidade pessoal e social em estudo prévio (Menéndez \& Fernández-Río, 2016). Outras intervenções mostraram melhora da lateralidade, do equilíbrio e da coordenação motora (Dinamond \& Lee, 2011). No aspecto cognitivo e socioafetivo, existem discussões pertinentes sobre a Educação Física escolar e a prática de AM (Correia, 2016; Vít \& Reguli, 2015), as quais indicam favorecer aspectos cognitivos, como a percepção, o raciocínio, a formulação de estratégias e a atenção. Já nos elementos socioafetivos, o comportamento apresenta maior coesão social em resposta as práticas próprias do wushu e da filosofia das AM (Cynarski \& Lee-Barron, 2014). Cabe apresentar uma limitação e uma sugestão para futuros estudos, o monitoramento das aulas de AM\&EC para verificar como as atividades de wushu são aplicadas nas escolas brasileiras e quais efeitos efetivos de cada uma dessas práticas.

Em resumo, as discussões sobre a presença das AM\&EC em meio escolar mostram que são prescritas, justificadas e sistematizadas a partir de abordagens particulares de ensino e definição que as situam como tema, conteúdo e linguagem da cultura corporal (Correia, 2016; Barros, 2011; Avelar \& Figueiredo, 2009; Ferreira, 2006; Pérez-Gutiérrez et al., , 2011). Diante disso, as aulas de wushu, podem ser adaptadas ao plano educacional escolar, como mostra o presente estudo com professores praticantes e não praticantes. Merecem recorte algumas formas de adaptar as práticas do wushu ao meio educacional, com atividades recreativas para aprendizagem de habilidades básicas, como empurrar, puxar, deslocar em duplas e representar as práticas competitivas através de jogos, como a luta do sapo (agachados, um tentando derrubar o outro) e a luta do saci (de mãos dadas, somente com um pé no chão, forçando o desequilíbrio do colega) (Ferreira, 2006). Parte-se do pressuposto que seja fundamental a preparação psicomotora de crianças e adolescentes através de aulas de lutas resignificadas no ensino fundamental, para práticas que envolvam manifestações filosóficas e éticas de maneira lúdica (Gomes, Barros, Freitas, \& Rufino, 2013). Tal pensamento já foi inclusive trazido à discussão e analisado dentro do universo marcial oriental por Theeboom et al. (2012), os quais também notaram uma menor preocupação com a transmissão de valores éticos, atualmente, com maior foco no âmbito ginástico e competitivo em si. 0 wushu, assim como as AM\&EC, em geral, também pode auxiliar o professor de educação física no desenvolvimento de conhecimentos no contexto histórico e social (Pérez-Gutiérrez, \& Gutiérrez García, 2012), já que para sua sobrevivência, o homem luta desde a pré-história (Franchini, 2014; Henning, 1997; Moreno, 2011, 2013). Por sua vez, as AM\&EC permitem abordar, de maneira educativa, essa condição humana.

\section{Considerações finais}

O presente trabalho investigou o wushu como Ferramenta para o Desenvolvimento Motor, Cognitivo e Socioafetivo na Escola e verificou as diferenças existentes em conceito, compreensão e na aplicação de práticas resignificadas para o desenvolvimento dos aspectos motores, cognitivos e socioafetivos no ensino fundamental I e II no Brasil. O objetivo desse estudo é discutir o wushu como ferramenta para o desenvolvimento motor, cognitivo e socioafetivo em crianças e adolescentes em meio escolar e verificar se existe diferença na compreensão e aplicação de práticas dessa AM no Ensino Fundamental I e II entre professores praticantes e não praticantes de wushu. Os dados mostraram diferença entre professores não praticantes e praticantes de wushu, sendo que os praticantes consideraram a modalidade um sistema válido para ensinar aspectos 
relacionados as artes marciais para escolares, enquanto o grupo de professores não praticantes acredita ser pouco eficiente. As demais questões apresentaram resultado similar entre os dois grupos. Não praticantes e praticantes reconheceram que o wushu é uma prática que atende as necessidades de desenvolvimento motor, cognitivo e socioafetivo em crianças e adolescentes, segundo as indicações dos Parâmetros Curriculares Nacionais. Segundo as indicações dos professores, as práticas de wushu superam ou satisfazem tais necessidades e raramente apresentam falhas no ensino-aprendizagem para escolares. 0 processo de ensino-aprendizagem do wushu é facilmente aplicado em meio escolar: todos os participantes do estudo responderam que recomendariam a utilização do wushu para escolares. Porém, cerca de $70 \%$ dos praticantes acreditam ser necessário realizar adaptações para ambientes escolares, enquanto metade dos não praticantes relatam não ser necessário fazer tais modificações para prática de wushu em ambiente escolar. Ao passo que grande parte dos entrevistados relatam que as informações sobre a prática de wushu durante a formação na graduação foram apenas parcialmente ou não foram realizadas de maneira clara. No seguimento exposto anteriormente, sugere-se que futuros estudos realizem entrevistas com professores universitários para verificar como ocorre a seleção das AM\&EC ensinadas no processo de graduação, sobre a preferência de professores sem experiência e também para verificar diferença entre gêneros.

\section{Referências}

Acevedo, W., Gutiérrez-García, C., \& Cheung, M. (2011). Breve história do Kung Fu. São Paulo: Madras.

Almeida, G. (2009). Teoria e prática em psicomotricidade: jogos, atividades lúdicas, expressão corporal e brincadeiras infantis. 6⿳亠丷厂 $\mathrm{ed}$. Rio de Janeiro: Wak.

Alves Junior, E. D. (2006). Discutindo a violência nos esportes de luta: a responsabilidade do professor de educação física na busca de novos significados para o uso das lutas como conteúdo pedagógico. Anais do XII Encontro Regional de História. Uso do passado. ANPUH, Arquivo Público do Estado do Rio de janeiro - APERJ.

Alves, F. (2012). Psicomotricidade: corpo, ação e emoção. Rio de Janeiro: Wak.

Amorim, G. P. (2004). Kung Fu um caminho para a saúde Física e Mental. São Paulo: Daemon Editora.

Ando, G. Y. U., Miarka, B., \& Pinto, M. M. (2016). Avaliação de análise técnico-tática em combates de Judô por programa computacional por usuários com diferentes níveis de expertise. Journal of Physical Education, 27(1), e2718.

Anjos, L. A. M., \& Moura, H. P. (2016). Um modelo para avaliação de produtos de software. Centro de Informática - Universidade Federal de Pernambuco (UFPE).

Associação Brasileira de Normas Técnicas - ABNT (2003). NBR ISO/IEC 9126-1: Qualidade de Produto - Parte 1: Modelo de qualidade. Rio de Janeiro: ABNT.

Avelar, B., \& Figueiredo, A. (2009). La iniciación a los deportes de combate: interpretación de la estructura del fenómeno lúdico luctatorio. Revista de Artes Marciales Asiáticas, 4(3), 44-57.

Avelar-Rosa, B., Gomes, M.S. P., Figueiredo, A., \& López-Ros, V. (2015). Caracterización y desarrollo del "saber luchar": contenidos de un modelo integrado para la enseñanza de las artes marciales y de los deportes de combate. Revista de Artes Marciales Asiáticas, 10(1), 16-33.

Barros, A. M., \& Gabriel, R. Z. (2011). Lutas. In S. C. Darido (Org.). Educação Física Escolar: compartilhando experiências. São Paulo: Phorte.

Brasil - Ministério da Educação (1997). Parâmetros Curriculares Nacionais: Educação Física / Secretaria de Educação Fundamental. Brasília: MEC/SEF.

Bu, B., Haijun, H., Yong, L., Chaohiu, Z., Xiaoyuan, Y., \& Singh, M. F. (2010). Effects of martial arts on health status: A systematicreview. Journal of Evidence-Based Medicine, 3(4), 205-219.

Bueno, M. O. (2012). Tai Chi Chuan e a Educação. Brasília: Jornal Lotus Bem Estar.

Correia, W. R., \& Franchini, E. (2010). Produção acadêmica em lutas, artes marciais e esportes de combate. Motriz, 16(1), 1-9.

Correia, W. R. (2016). Educação Física Escolar e Artes Marciais: entre o combate e o debate. Revista Brasileira de Educação Física e Esporte, 29(2), 337-344.

Cynarski, W. J., \& Lee-Barron, J. (2014). Philosophies of martial arts and their pedagogical consequences. Ido Movement for Culture, 14(1), 11-19. 
Cynarski, W. J., Pawelec, P., Zeng, H. Z., Yu, J. H., Vit, M., Bielec, G., Slopecki, J., Kubala, K., \& Blazejewski, W. (2017). Social Determinants of Attitudes towards Health in Martial Arts: comparison between combat sports and combat systems and martial arts practitioners. Ido Movement for Culture, 17(1), 23-29.

Cynarski, W. J., \& Skowron J. (2014). An analysis of the conceptual language used for the general theory of martial arts - Japanese, Polish and English terminology. Ido Movement for Culture,. 14(3), 49-66.

Del Vecchio, F. B., \& Franchini, E. (2006). Lutas, artes marciais e esportes de combate: possibilidades, experiências e abordagens no currículo em educação física. In S. Souza Neto \& D. Hunger (Orgs.). Formação profissional em educação física: estudos e pesquisas. Rio Claro: Biblioética.

Del Vecchio, F., \& Franchini, E. (2012). Princípios pedagógicos e metodológicos das Lutas. In E. Franchini \& F. B. Del Vecchio. (Org.). Ensino de Lutas: Reflexões e Propostas de Programas (pp. 9-27). São Paulo: Scortecci Editora.

Di Zio S. (2010). Future strategy for reducing violence against women: The Italian experience. Foresight, 12(5), 80-91.

Diamond, A., \& Lee, K. (2011). Intervention shown to aid executive function development in children 4 to 12 years old. Science, 333, 959-964.

Donohue, J.J. (2005). Modern educational theories and traditional japanese martial arts training method. Journal of Asian Martial Arts, 14(2), 8-29.

Durántez, C. (2004). Los deportes de lucha en el calendario competitivo de Olimpia. Dimensión Histórica, Cultural y Deportiva de las Luchas (pp. 13-32). Fuerteventura: Asociación CientíficoCultural para el Estudio de los Deportes de Lucha (ACCEDEL).

Faculdade Einstein, FACEI. (2015). Curso de Especialização em Shuai Jiao (Extensão). São Paulo: Faculdade Einstein - FACEI.

Faculdade Einstein, FACEI. (2016). Metodologia e didática do ensino da Educação Física e das Artes Marciais. São Paulo: Faculdade Einstein.

Ferreira, H. S. (2006). As Lutas na Educação Física Escolar. Revista de Educação Física, 135, 36-44.

Figueiredo, A. (1997). Os Desportos de Combate nos Programas de Educação Física. Horizonte, 80, 36-39.

Figueiredo, A. (1998). Os Desportos de Combate nas Aulas de Educação Física. Horizonte, 81, Dossier.

Figueiredo, A. (2000). Os Desportos de Combate nas Aulas de Educação Física. $8^{o}$ Congresso deEducação Física e Ciências do Desporto dos Países de Língua Portuguesa. 16-17 dezembro 2000. Lisboa: Faculdade de Motricidade Humana, Universidade Técnica de Lisboa. Disponível em http://repositorio.ipv.pt/handle/10400.19/465

Figueiredo, A. (2006). A Institucionalização do Karaté - Os Modelos Organizacionais do Karaté em Portugal. Tese de Doutoramento (não publicada). Lisboa: Faculdade de Motricidade Humana, Universidade Técnica de Lisboa.

Franchini, E. (2014). Born to fight - Genetics and combat sports. Revista de Artes Marciales Asiáticas, 9(1), 1-8.

Franchini, E., \& Del Vecchio, F.B. (2012). Ensino das lutas: Reflexões e Propostas de Programas. Scortecci: São Paulo.

Gomes, M. S. P. (2008). Procedimentos pedagógicos para o ensino das lutas: contextos e possibilidades. Dissertação de mestrado apresentada à Pós-Graduação da Faculdade de Educação Física da Universidade Estadual de Campinas para obtenção do título de mestre em Educação Física. Campinas.

Gomes, M., \& Avelar-Rosa, B. (2012). Martial Arts and Combat Sports in Physical Education and Sport Sciences Degrees - A comparative study of Brazil, France, Portugal and Spain. InYo The Journal of Alternative Perspectives on the Martial Arts and Sciences, 12(1), 13-28.

Gomes, N., de Barros, A., de Freitas, F., Darido, S., \& Rufino, L. (2013). 0 conteúdo das lutas nas séries iniciais do ensino fundamental: possibilidades para a prática pedagógica da Educação Física escolar. Motrivivência, (41), 305-320.

Henning, S. (1997). Chinese boxing: The internal versus external school in the light of history and theory. Journal of Asian Martial Arts, 6(3), 10-19. 
Hortiguela, D., Gutiérrez-García, C., \& Hernando-Garijo, A. (2017). Combat versus team sports: the effects of gender in a climate of peer-motivation, and levels of fun and violence in physical education students. Ido Movement for Culture, 17(3), 11-20.

IWUF. (2012). IWUF Presentation to the IOC Olympic Programme Commission. Disponível em http://www.cbkw.org.br/novo/wp-content/uploads/2012/12/Press-Release LausannePresentation 2012-wushu.pdf

IWUF. (2015). IWUF Raises wushu's Profile at SportAccord Convention in Sochi. Disponível em http://www.iwuf.org/upload/2015/0518/ba48349eff31cf6137a7ec63307d82a0ff7c2749.p $\underline{\mathrm{df}}$

Johnson, J. A., \& Ha, P. (2015). Elucidating pedagogical objectives for combat systems, martial arts, and combat sports. Ido Movement for Culture, 15(4), 65-74.

Kusnierz, C., Cynarski, W. J., \& Gorner, K. (2017). Social reception and understanding of combat sports and martial arts by both school students and adults. Ido Movement for Culture, 17(1), 30-37.

Light, R. (2014). Mushin and learning in and beyond Budo. Ido Movement for Culture, 14(3), 42-48.

Lima, A. (1997). Os Desportos de Combate (DC) como matéria de Educação Física (EF) nos Ensino Básico e Secundário. Horizonte, 86, 17-28.

Lima, L. M. S. (2000). O Tao da Educação: a Filosofia Oriental na escola Ocidental. São Paulo: Agora.

Lima, M. (2012). A Emergência do Ensino dos Desportos de Combate nas Aulas de Educação Física. Dissertação com vista à obtenção do Título de Mestre (não publicada). Vila Real: Universidade de Trás-os-Montes e Alto Douro.

Martin, B. (2015). Report on the 4th International Martial Arts and Combat Sports Scientific Society (IMACSSS) Symposium. Revista de Artes Marciales Asiáticas, 10(2), 135-137.

Martins, A. T. (2011). Processo Ensino-aprendizagem em Deficientes Intelectuais: Uma Proposta de Intervenção Através do Kung Fu. In VII Encontro da Associação Brasileira de Pesquisadores em Educação Especial, Londrina.

Menéndez J.I., \& Fernández-Río J. (2016). Violence, Responsibility, Friendship and Basic Psychological Needs: Effects of a Sport Education and Teaching for Personal and Social Responsibility Program. Journal of Psychodidactics, 21(2), 245-260.

Metzler M. (2011). Instructional models for physical education. Scottsdale: Hawthorne Hathaway.

Miarka B., \& Conceição R. (2012). Pedagogia do ensino da luta olímpica em escolas. In E. Franchini \& F.B. Del Vecchio (Eds.), Ensino das lutas: Reflexões e Propostas de Programas (pp. 183-208). Scortecci: São Paulo.

Miarka, B., Marques, J. B., \& Franchini, E. (2011). Reinterpreting the history of women's judo in Japan. International Journal of History Sport, 28, 1016-1029.

Millán, G. A. (2015). El Dojang: escuela de disciplina y moralidad. Revista de Artes Marciales Asiáticas, 10(1), 1-15.

Mocarzel, R. C. S. (2011). Artes marciais e jovens: violência ou valores educacionais? Um estudo de caso de um estilo de Kung-Fu. Dissertação apresentada para obtenção do Grau de Mestre em Ciências da Atividade Física - Universidade Salgado de Oliveira. Niterói.

Mocarzel, R. C. S., \& Murad, M. (2013). O não uso das lutas na educação física escolar brasileira. In R. Osborne, C. A. F. Silva, \& R. F. Santos. (Org.). Complexidade da educação física escolar. Rio de Janeiro: Lamparina.

Mocarzel, R., Ferreira, A. S., \& Murad, M. (2014). Comparação dos motivos de ingresso e de permanência de jovens praticantes nas aulas de Kung-Fu. Corpus et Scientia, 10(2), 63-72.

Moreno, E. (2011). The society of our "out of Africa" ancestors (I) - the migrant warriors that colonized the world. Communicative \& Integrative Biology, 4(2), 163-170.

Moreno, E. (2013). The "out of Africa tribe" (II) - paleolithic warriors with big canoes and protective weapons. Communicative \& Integrative Biology, 6(3), e24145.

Nascimento, P., \& Almeida, L. (2007). A tematização das Lutas na Educação Física escolar: restrições e possibilidades. Movimento, 13(3), 91-110.

Ortega, E. M., \& Antunes, M. M. (2013). O Ensino das Artes Marciais para Crianças e Adolescentes na Perspectiva da Saúde, In M. M. Antunes \& C. C. Iwanaga (Orgs.). Aspectos Multidisciplinares das Artes Marciais. Jundiaí: Paco Editorial. 
Pérez-Gutiérrez, M., \& Gutiérrez García, C. (2012). Estudo bibliográfico sobre as monografias das artes marciais publicadas em Espanha (1906-2006). Revista de Artes Marciales Asiáticas, 3(4), 22-33.

Pérez-Gutiérrez, M., Gutiérrez-García, C., \& Escobar-Molina, R. (2011). Terminological recommendations for improving the visibility of scientific literature on martial arts and combat sports. Archives of Budo, 7(3), 159-166.

Robles, J. (2008). Causas de la escasa presencia de los deportes de lucha con agarre en las clases de Educación Física en la ESO. Retos - Nuevas Tendencias en Educación Física, Deporte y Recreación, 14, 43-47.

Rufino, L. G. B., \& Darido, S. C. (2015). O ensino das lutas nas aulas de Educação Física: análise da prática pedagógica à luz de especialistas, Revista da Educação Física, 26(4), 505-518.

Saeki, T. (1994). The conflict between tradition and modernization in a sport organization: a sociological study of issues surrounding the organizational reformation of the all Japan Judo Federation. International Review for the Sociology of Sport, 29(3), 301-315.

Sasaki, T. (2006). The meaning and role of budo (the martial arts) in school education in Japan. Archives of Budo, 2(1), 11-14.

Tarabanov, A. (2011). Therapeutic ethos and martial arts. Ido Movement for Culture, 11(1), 33-36.

Tejero-González, C. M., Balsalobre-Fernández, C., \& Ibáñez-Cano, A. (2011). La defensa personal como intervención educativa en la modificación de actitudes violentas. Revista Internacional de Medicina y Ciencias de la Actividad Física y del Deporte, 11(43), 513-530.

Theeboom M., \& De Knop P. (1999). Asian Martial Arts and Approaches of Instruction in Physical Education. European Journal of Physical Education, 4(2), 146-161.

Theeboom M., De Knop P., \& Weiss M.R. (1995). Motivational Climate, Psychological Responses, and Motor Skill Development in Children's Sport: A Field-Based Intervention Study. Journal of Sport \& Exercise Psychology, 17, 294-391.

Theeboom, M., Dong, Z., \&Vertonghen, J. (2012). Traditional Asian martial arts and youth: Experiences of young Chinese wushu athletes. Archives of Budo, 8(1), 27-35.

Theeboom, M., De Knop, P., \& Vertonghen J. (2009). Experiences of children in martial arts. European Journal for Sport and Society, 6(1), 19-35.

Thomas, J.R., \& Nelson, J.K. (2002). Métodos de pesquisa em atividade física. 3 ed. Artmed: Porto Alegre.

Torres, J. A. M. (2011). Kung Fu: a milenar arte marcial chinesa: águia, bêbado, louva-a-deus, tai chi chuan, tigre, wing chun. São Paulo: Online Editora.

Universidade de São Paulo - USP. (2016). Práticas Corporais da Medicina Tradicional Chinesa. Foco em Tai Chi Chuan da Família Yang e sua Filosofia. Escola de Artes, Ciências e Humanidades. Universidade de São Paulo - USP. Disponível em: https://uspdigital.usp.br/apolo/apoObterCurso?cod curso $=860100010 \& \operatorname{cod}$ edicao $=15001$ \&numseqofeedi $=1$

Universidade Estadual Paulista Júlio Mesquita Filho - UNESP. (2016). Especialização em Lutas e Artes Marciais. Faculdade de Ciências - Universidade Estadual Paulista Júlio Mesquita Filho UNESP - Campus de Bauru. Disponível em http://www.fc.unesp.br/\#!/posgraduacao/especializacao/lutas-e-artes-marciais

Universidade Gama Filho - UGF. (2011). Lutas e artes marciais: da pedagogia ao treinamento. Universidade Gama Filho - UGF. Disponível em http://www.posugf.com.br/cursos/posgraduacao/educacao-fisica/1711-lutas-e-artes-marciais-da-pedagogia-ao-treinamento

Verthonghen, J., \& Theeboom, M. (2010). The Social-Psychological outcomes of Martial Arts practise among young: a review. Journal of Sports Science and Medicine, 9, 528-537.

Villamón, M., Gutiérrez-García, C., Espartero, J., \& Molina, J.P. (2005). La práctica de los deportes de lucha: un estudio preliminar sobre la experiencia previa de los estudiantes de la Licenciatura en Ciencias del Deporte. Apunts: Educación física y deportes, 79, 13-19.

Vít, M., \& Reguli, Z. (2015). The role of combatives teaching in physical education. Brno: Faculty of Sports Studies, Masaryk University. Disponível em https://munispace.muni.cz/index.php/munispace/catalog/book/694

Weng, C. D. (1990). Fundaments of Shuai Chiao. 2ª ed. Taipei - ROC: Chinese Culture University. 
Zivin, G., Hassan, N. R., De Paula, G. F., Monti, D. A., Harlan, C., Hossain, K. D., \& Patterson, K. (2001). An effective approach to violence prevention: Traditional martial arts in middle school. Adolescence, 36(143), 443-459.

\section{Author's biographical data}

Everton de Souza da Silva. DA SILVA, E. S. (Brasil). Graduado em Educação Física - Licenciatura (UNIBAN); Pós-graduado em Defesa Pessoal Aplicada à Segurança Pública e Privada com Ênfase em Shuaijiao (FACEI), Graduando em Esporte (EEFE/USP). Atleta da Seleção Brasileira de Shuaijiao (2013 e 2015) e Faixa Preta 1o Grau (9 deng) de Baoding Shuaijiao. E-mail: evertonsilva@usp.br

Diego Pereira Alves. ALVES, D. P. (Brasil). Graduado em Educação Física - Licenciatura (UNIBAN); Graduando em Educação Física - Bacharelado (UNIAN). E-mail: diegodparhcp@hotmail.com

Lucas Ribeiro dos Santos. DOS SANTOS, L. R. (Brasil). Graduado em Educação Física - Licenciatura (UNIBAN). E-mail: lucas ribeiro45@hotmail.com

Marcelo Augusto Pinto dos Santos. SANTOS, M. A. P. (Brasil). Graduado em Educação Física - Licenciatura (UNIBAN). E-mail: marceloaugusto123@gmail.com

Sumara Brito Soares. SOARES, S. B. (Brasil). Graduada em Educação Física - Licenciatura (UNIBAN); Graduando em Educação Física - Bacharelado (UNIAN).E-mail: sumarabsoares@gmail.com

Bianca Miarka. MIARKA, B. (Brasil). Professora do Departamento de Educação Física da Universidade Federal de Juiz de Fora, Campus Governador Valadares (UFJF/GV). doutora em Biodinâmica (EEFE/USP), mestre em Educação Física (EEFE/USP) e graduada em Ciência do Esporte (UEL). E-mail: bianca.miarka@uffif.edu.br 